\title{
COMPARACIÓN ENTRE LAS BIOPSIAS GÁSTRICAS SIN FIJAR 24 HORAS FRENTE A LA BIOPSIA CONVENCIONAL PARA EL DIAGNÓSTICO DE Helicobacter pylori EN UN HOSPITAL DE REFERENCIA DE PERÚ
}

\author{
César Tadeo Vela-Velásquez $1, a, b$
}

\begin{abstract}
RESUMEN
Objetivos. Comparar biopsias gástricas fijadas inmediatamente con formol al 10\% frente a biopsias fijadas tras 24 horas en la detección de Helicobacter pylori $(\mathrm{Hp})$ en un hospital de referencia en Perú. Materiales y métodos. Se realizó un estudio de test diagnóstico sobre las biopsias gástricas de 72 pacientes que acudieron al Servicio de Gastroenterología del Hospital Essalud Alberto Sabogal en el Callao. Las muestras de cada paciente fueron repartidas en dos frascos de vidrio, una de ellas se fijó inmediatamente con formol al 10\% y a la otra se fijó luego de 24 horas. Se procesaron ambos grupos de muestras con la técnica convencional de inclusión en parafina y coloración con hematoxilina-eosina. Resultados. Se evidenció Hp en 56,9\% del grupo de biopsias fijadas inmediatamente y en el 79,2\% del grupo de biopsias fijadas tras 24 horas, encontrándose diferencia estadística $(p<0,001)$. Conclusiones. Existe una mayor frecuencia de diagnóstico de $\mathrm{Hp}$ en las muestras que se fijaron luego de 24 horas; lo que podría sugerir que ello actuaría como un cultivo biológico para Hp que facilitaría su diagnóstico.
\end{abstract}

Palabras clave: Helicobacter pylori; Técnicas de Diagnóstico del Sistema Digestivo; Biopsia, Patología Clínica; Perú (Fuente: DECS BIREME).

\section{COMPARISON BETWEEN GASTRIC BIOPSIES WITHOUT FIXATION FOR 24 HOURS AND CONVENTIONAL BIOPSY FOR THE DIAGNOSIS OF Helicobacter pylori IN A REFERENCE HOSPITAL IN PERU}

\begin{abstract}
Objectives. To compare gastric biopsies immediately fixed with $10 \%$ formalin with biopsies fixed after 24 hours for the detection of Helicobacter pylori (Hp) in a reference hospital in Peru. Materials and methods. A diagnostic test study on gastric biopsies from 72 patients attending the Gastroenterology service of hospital Essalud Alberto Sabogal in Callao was performed. Samples from each patient were divided into two glass jars, one of which was fixed immediately with $10 \%$ formalin and the other was fixed after 24 hours. Both groups of samples were processed with the conventional technique of paraffin embedding and staining with hematoxylin-eosin. Results. Hp was found in $56.9 \%$ of the group of biopsies which were inmediatly fixed and in $79.2 \%$ of the group of biopsies fixed after 24 hours, showing statistical difference $(p<0.001)$. Conclusions. There is an increased frequency of diagnosis of $\mathrm{Hp}$ in the samples that were fixed after 24 hours, which may suggest that this would act as a biological culture for $\mathrm{Hp}$, which would facilitate its diagnosis.
\end{abstract}

Key words: Helicobacter pylori; Diagnostic Techniques, Digestive System; Pathology, Clinical; Peru (Source: MESH NLM).

\section{INTRODUCCIÓN}

A diferencia de los primeros reportes sobre Helicobacter pylori $(\mathrm{Hp})^{(1)}$ en la década de 1980, en la actualidad se conoce mucho más sobre esta bacteria; se le relaciona en la génesis de úlcera péptica, linfomas y neoplasias gástricas como el adenocarcinoma (2-7). Ha sido considerado por la Organización Mundial de la Salud como agente carcinógeno tipo ${ }^{(8)}$.
Para el diagnóstico de Hp se ha utilizado métodos histológicos, cultivos microbiológicos, serológicos, prueba de la ureasa, pruebas de antígenos en heces, pruebas moleculares de ADN y ARN (9-14), e incluso combinaciones de estos métodos ${ }^{(15,16)}$. Actualmente, el diagnóstico definitivo se basa en el aislamiento del $\mathrm{Hp}$ en las biopsias gástricas y en los cultivos microbiológicos, sin embargo, estos últimos requieren medios especiales y ambientes controlados, por lo que la mayoría de laboratorios no

1 Médico Patólogo.

a Servicio de Anatomía Patológica del Hospital Essalud "Alberto Sabogal Sologuren".

b Director Científico del Instituto de Investigación de Citopatología-CITOPAT. 
pueden realizarlos. En los estudios histológicos se utiliza generalmente la coloración de hematoxilina-eosina (HE), pero esta coloración tiene una menor sensibilidad para la detección de Hp que la coloración de Warthin Starry, la cual es de mayor costo y requiere un personal técnico especializado, por lo que en los servicios de anatomía patológica se realiza esporádicamente ${ }^{(9-16)}$.

Considerando ese importante factor de factibilidad expuesto en el párrafo previo, se buscaría obtener métodos con mejores propiedades diagnósticas pero que ello no implique un incremento considerable de demanda de recursos económicos; por lo que mantener la coloración HE sería importante siempre y cuando se logre que la población de bacterias viables en la biopsia sea más elevada que la que se obtiene con la fijación inmediata.

Es preciso mencionar, que llegó a nuestro servicio de patología una biopsia gástrica accidentalmente sin fijar habitualmente tiene que llegar en un medio de fijación, generalmente con formol al $10 \%$, de no ser así el proceso de necrobiosis, dependiendo del tiempo, puede inutilizar dicha muestra para el estudio histológico- en la cual, por medio de la microscopía se observaron colonias de $\mathrm{Hp}$, hallazgo inédito, puesto que en las biopsias convencionales muchas veces se encuentra abundante cantidad de la bacteria pero jamás se llegan a formar colonias.

Por lo anterior, supondríamos que al ser extraída la muestra para biopsia, la respuesta inmunológica se hace nula y el $\mathrm{pH}$ al no producirse el ácido clorhídrico se altera, con lo que existirian las condiciones para que si hay $\mathrm{Hp}$, esta población se haga más numerosa, es decir, existiría un aprovechamiento de la presencia de nutrientes existentes en el epitelio foveolar de las glándulas gástricas y en ausencia de elementos que impiden su crecimiento, daría como resultante el aumento de bacterias, con lo que la biopsia gástrica sin fijar 24 horas actuaría como un cultivo biológico.

Con este antecedente se llevó a cabo el presente estudio con el objetivo primario de comparar las biopsias convencionales fijadas inmediatamente (BF) con formol al 10\% frente a las biopsias fijadas tras 24 horas (BSF) en la detección de Helicobacter pylori $(\mathrm{Hp})$ en un hospital de referencia en Perú. Asimismo, se buscó evaluar si la falta de fijación de 24 horas altera la nitidez y el grosor del corte histológico imposibilitando la correcta lectura.

\section{MATERIALES Y MÉTODOS}

\section{DISEÑO DE ESTUDIO}

Se desarrolló un estudio prospectivo de corte transversal tipo test diagnóstico.

\section{POBLACIÓN DE ESTUDIO}

Con un nivel de significancia de $5 \%$, para un estudio de dos colas, estimando una proporción de éxito en el mejor de los casos de 80\%; un índice kappa esperado de 0,5 entre los dos métodos y un nivel de 0,14 para encontrar diferencias entre los niveles y una potencia de $80 \%$, se requería el mínimo de 69 pacientes.

Se incluyeron 72 pacientes atendidos en el Servicio de Gastroenterología del Hospital "Alberto Sabogal Sologuren" en la ciudad de Callao, durante los meses de junio y julio de 2007. Estos pacientes no presentaron hallazgosmacroscópicosanormalesdurantelaendoscopía alta y la muestra para el estudio anatomopatológico fue exclusivamente para el estudio por $\mathrm{Hp}$. Se excluyó a las personas con lesiones tumorales observadas en la endoscopía alta, con lesiones malignas precoces, aquellos que venían para su control endoscópico posterior al tratamiento de erradicación de $\mathrm{Hp}$, asimismo, las muestras obtenidas en endoscopía de emergencia.

\section{TOMA DE MUESTRA Y ASIGNACIÓN DE LAS} EVALUACIONES ANATOMOPATOLÓGICAS

Las biopsias obtenidas se repartieron en dos frascos de vidrio, una de ellas se llenaba con formol al $10 \%$ y la otra sin formol. Las muestras con formol fueron enviadas al Servicio de Patología para el procedimiento habitual. La evaluación fue ciega dado que los observadores (cuatro médicos patólogos) no sabían que formaban parte de un estudio de investigación.

Las muestras sin fijar fueron remitidas a uno de los patólogos investigadores y se mantuvieron sin fijación durante 24 horas. Posteriormente se les agregó formol al $10 \%$ para la fijación habitual y ser procesadas con la técnica convencional de inclusión en parafina y coloración con hematoxilina-eosina. Para la lectura de láminas, se remitieron a los patólogos sin que ellos tengan información sobre la procedencia de dichas biopsias.

\section{TÉCNICA DE OBSERVACIÓN Y PRUEBA DE CONTROL}

En las láminas ya coloreadas se buscaron (con aumento de 400X y en casos de duda se utilizó aumento de 1000X con aceite de inmersión) bacilos con morfología de Hp. Para su cuantificación se procedió a ubicar el área de mayor población bacteriana y se clasificó del siguiente modo:
0 :

10 "+":

20 "++":
Ausencia de $\mathrm{Hp}$

1-20 Hp en campo de 400X

21-100 Hp en campo de 400X 
3 o "+++": $\quad$ >100 Hp en campo de 400X (sin llegar a ser colonia)

4: Colonias de Hp en campo de 400X

Como prueba de control, se tomó al azar tacos de parafina de cinco pacientes cuyos resultados fueron negativos para $\mathrm{Hp}$ en las BF y positivo en las BSF. Luego se procedió a hacer nuevos cortes que fueron coloreados con la técnica de Warthin Starry que tiene alta especificidad y sensibilidad ${ }^{(17)}$, y las diez láminas fueron entregadas a uno de los investigadores (quién no sabía a cual grupo correspondía la lámina que estaba estudiando) para el diagnóstico del Hp.

\section{EVALUACIÓN DE LA FIJACIÓN}

Para evaluar si la falta de fijación por 24 horas alteró la estructura microscópica del tejido, observamos la nitidez y el grosor del corte histológico en las BF y en las BSF, por lo que las láminas histológicas fueron mezcladas al azar y uno de los investigadores las evaluó sin saber a que grupo pertenecían con la siguiente cuantificación:

1: Inadecuado para el estudio histológico

2: Adecuado para el estudio histológico

\section{ANÁLISIS ESTADÍSTICO}

Se utilizó el programa estadístico SPSS 15.0 para evaluar la significancia estadística con la Prueba $T$ de Wilcoxon para dos muestras relacionadas y la prueba de Mc Nemar para muestras relacionadas. También se empleó el coeficiente de concordancia de Kappa.

\section{ASPECTOS ÉTICOS}

A pesar que rutinariamente se toman dos o tres biopsias gástricas para la detección de Hp, se contó con el consentimiento informado de los pacientes. El documento, elaborado en coordinación entre los Servicios de Gastroenterología y Anatomía Patológica, explicaba que la biopsia para el estudio se utilizaría únicamente para determinar la presencia de $\mathrm{Hp}$, que una parte de la muestra se fijaba inmediatamente como se hace de rutina y la otra parte se fijaba después de 24 horas.

\section{RESULTADOS}

\section{CARACTERÍSTICAS GENERALES}

La edad promedio de los 72 pacientes fue de 48,3 \pm 15,5 años; $51(70,8 \%)$ fueron mujeres con una edad media de 47,6 \pm 14,9 años, y 21 (29,2\%) fueron varones con una edad media de 50,1 $\pm 16,9$ años.
Tabla 1. Distribución de la cuantificación de Helicobacter pylori en la población estudiada de acuerdo con la técnica de fijación usada.

\begin{tabular}{ccc}
\hline $\begin{array}{c}\text { Número } \\
\text { de } \mathrm{Hp}\end{array}$ & $\begin{array}{c}\text { Biopsias } \\
\text { convencionales } \mathbf{N}(\%)\end{array}$ & $\begin{array}{c}\text { Biopsias sin fijar } \\
\mathbf{2 4} \text { horas } \mathbf{N}(\%)\end{array}$ \\
\hline 0 & $31(43,1)$ & $15(20,8)$ \\
1 a 20 & $23(31,9)$ & $30(41,7)$ \\
21 a 100 & $15(20,8)$ & $13(18,1)$ \\
> 100 & $3(4,2)$ & $9(12,5)$ \\
Colonias & $0(0)$ & $5(6,9)$ \\
\hline
\end{tabular}

\section{NÚMERO DE BIOPSIAS}

El número de biopsias por paciente en el grupo de BF fue: 33 con una biopsia, 37 con dos biopsias y 2 con tres biopsias. El número de biopsias por paciente en el grupo de BSF fue: 44 con una biopsia, 26 con dos biopsias y 2 con tres biopsias. No hubo diferencia estadística en el número de biopsias entre ambos grupos ( $p>0.05)$. Todas las biopsias estudiadas fueron catalogadas como adecuadas para el estudio anatomopatológico.

\section{DETECCIÓN DE Helicobacter pylori}

A diferencia de lo encontrado en las biopsias procesadas en forma convencional, en el grupo sin fijar por 24 horas se encontró prácticamente en todos los casos mayor número de $\mathrm{Hp}$, incluso se reportó la formación de colonias en cinco de ellas (Tabla 1).

Como puede observarse en la Tabla 2, existe diferencia estadística en la detección de biopsias entre la primera y segunda técnica. $(p<0,001)$. Asimismo, en esta tabla se presenta el análisis del coeficiente kappa, donde se observa una fuerza de concordancia moderada.

En la Figura 1 observamos una muestra BF positiva para $\mathrm{Hp}$ en cuyo equivalente BSF se distinguieron colonias de Hp. De 16 muestras BF negativas para Hp y positivas para BSF, se tomaron al azar cinco para teñirlas con la técnica de Warthin Starry, encontrándose positividad en tres del grupo de BF y en los cinco del grupo con BSF.

La Figura 2 se muestra Hp la comparación entre una biopsia BSF coloreada con hematoxilina-eosina y la tinción de de Warthin Starry.

Tabla 2. Detección de Helicobacter pylori en la población en estudio mediante fijación con formol al $10 \%$ de manera inmediata y luego de 24 horas.

\begin{tabular}{ccccc}
\hline & $\begin{array}{c}\text { Biopsias } \\
\text { Convencionales } \\
\mathbf{N}(\%)\end{array}$ & $\begin{array}{c}\text { Biopsias sin } \\
\text { fijar 24 horas Kappa }\end{array}$ & $\mathbf{p}$ \\
& $\mathbf{N}(\%)$ & & \\
\hline PRESENTE & $41(56,9)$ & $57(79,2)$ & 0,516 & $<0,001$ \\
\hline AUSENTE & $31(43,1)$ & $15(20,8)$ & & \\
\hline
\end{tabular}



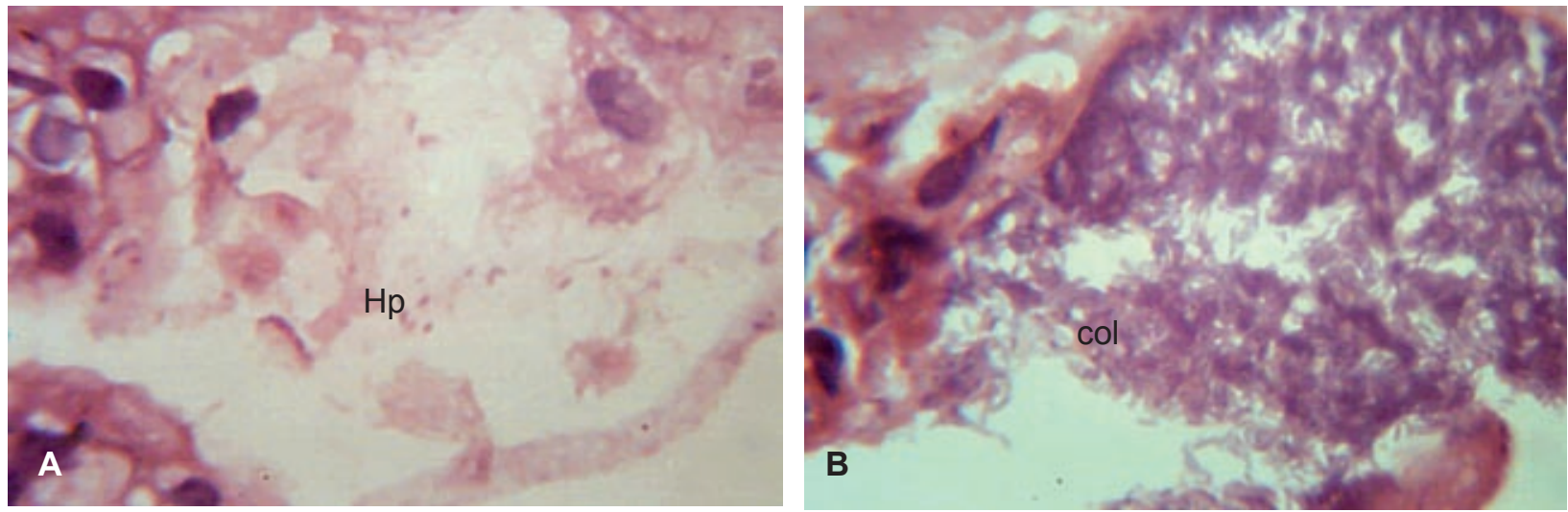

Figura 1. A Biopsia convencional mostrando $\mathrm{Hp}$ en el epitelio glandular.1000X. B Biopsia sin fijación de 24 horas mostrando una colonia (col) de Hp. Coloración de Hematoxilina-Eosina. 400X.

\section{DISCUSIÓN}

La proporción de casos detectados de Hp en el grupo de BF $(56,9 \%$ positivos) tiene similitud con investigaciones nacionales como el estudio de Ramírez et al. ${ }^{(18)}$ quienes en un grupo de 1406 pacientes con características clínicas similares a nuestra población de estudio, encontraron $57,3 \%$ de positividad; además, es similar con los resultados de Rivera et al. ${ }^{(19)}$ en México, donde estudiaron 151 pacientes y el $57,24 \%$ tuvieron cultivo positivo para Hp. Sin embargo, en la presente investigación al utilizar la BSF la detección fue superior $(79,2 \%)$.

La validez del método descrito, se refuerza por los resultados obtenidos en las muestras de los pacientes negativos en BF y positivos en sus respectivas BSF, donde la coloración de Warthin-Starry fue positiva en más de la mitad $(60 \%)$ de las biopsias del grupo BF y en la totalidad del grupo BSF correspondiente. Por lo que estos hallazgos podrían sugerir, aunque de manera indirecta, que habría un subdiagnóstico al usar solamente el BF; por lo que en un caso hipotético podríamos suponer que habría un incremento de pacientes diagnosticados correspondiente al $60 \%$ de los pacientes negativos con BF al usarse la técnica Warthin-Starry, es decir, ligeramente superior al $70 \%$; no obstante, ello es inferior al 79,2\% logrado con la BSF.

En ese sentido, Velapatiño et al. ${ }^{(20)}$ realizaron la validación del entero test para el diagnóstico de la infección por $\mathrm{Hp}$ en 44 pacientes peruanos, considerando como prueba patrón la positividad de 35 pacientes $(79,5 \%)$ medido por biopsia y cultivo de $\mathrm{Hp}$ (sumando las positividades parciales), lo que es muy similar a nuestra detección con las $\operatorname{BSF}(79,2 \%)$.

Lo expuesto en los párrafos previos, implicaría que la única diferencia probable sería el procesamiento de las biopsias de los dos grupos, la cual tuvo implicaciones sobre la alteración la presencia de $\mathrm{Hp}$ en el epitelio glandular gástrico favoreciendo su crecimiento, por lo que daría la suposición, que la biopsia sin fijar actuaría como un medio de cultivo biológico para esta bacteria. No se han encontrado antecedentes en la literatura científica, constituyendo el primer reporte de este método de ayuda diagnóstica a nivel mundial.
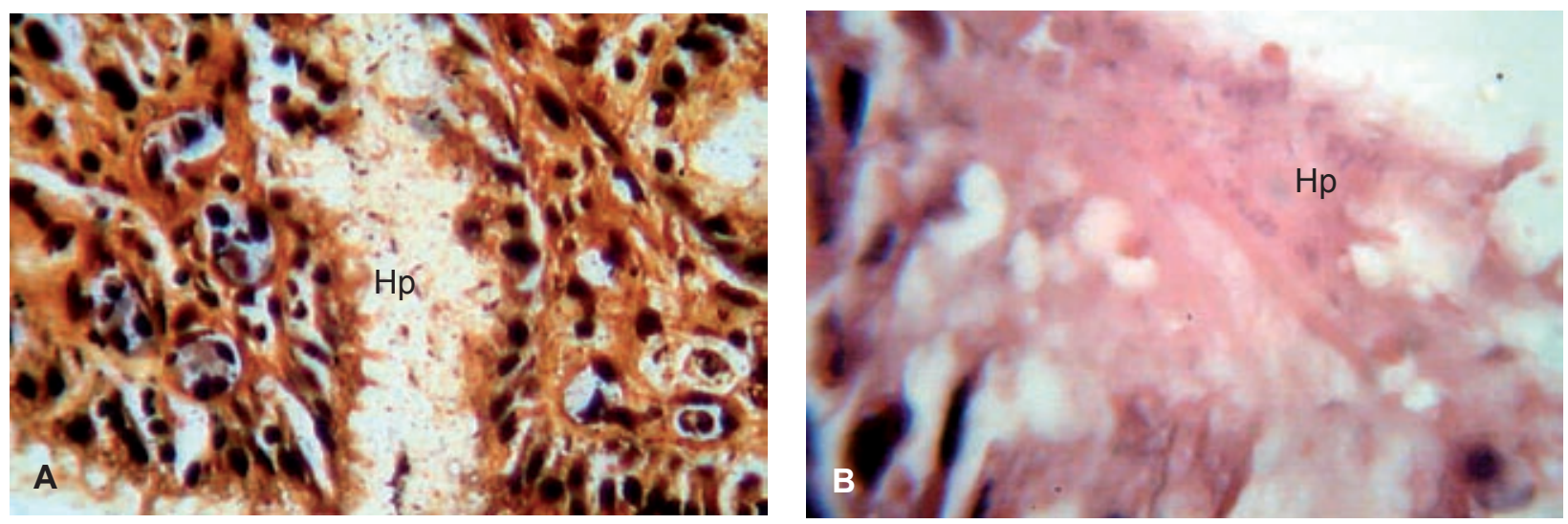

Figura 2. A. Positividad para Hp con la coloración de Warthin Starry. B. Positividad para Hp con hematoxilina-eosina (400X). 
Es preciso mencionar que el estudio presenta algunas limitaciones: podría existir una variación entre los observadores que no ha sido medida. Asimismo, el tamaño muestral no fue calculado para evaluar la técnica de Warthin-Starry. Por otro lado, el método que describimos como cultivo biológico, todavía no se puede aplicar para la obtención del antibiograma, aspecto que tendría alta importancia en las decisiones clínicas que pueda tomarse.

Finalmente consideramos que la biopsia sin fijación inmediata requiere investigaciones y evaluaciones posteriores con tamaños de muestra mayores que permitan detectar mínimas diferencias. Si las investigaciones posteriores confirman los hallazgos remitidos en el presente artículo; esta técnica podría convertirse en examen auxiliar de referencia para el diagnóstico de $\mathrm{Hp}$.

\section{AGRADECIMIENTOS}

Al Dr. Guillermo Huarcaya por el apoyo en la toma de muestras.

\section{Fuentes de Financiamiento \\ Autofinanciado.}

\section{Conflictos de Interés}

El autor declara no tener conflictos de interés en la publicación de este artículo.

\section{REFERENCIAS BIBLIOGRÁFICAS}

1. Marshall B, Warren R. Unidentified curved bacilli in the stomach of patients with gastritis and peptic ulceration. Lancet 1984;1(8390):1311-5.

2. Blecker U, McKeithan TW, Hart J, Kirschner BS. Resolution of Helicobacter pylori-associated gastric lymphoproliferative disease in a child. Gastroenterology. 1995;109(3):973-7.

3. Graham D. Helicobacter pylori infection in the patogénesis of ulcer and gastric cancer a model. Gastroenterology. 1997; 113(6): 1983-91.

4. McColl KE, El-Omar RE, Guillén D. The role of Helicobvacter pylori in the pathophysiology of duodenal Ulcer and gastric cancer. Semin Gastrointest. Disease. 1997;8(3):142-55.

5. Oberhuber G, Kranz A, Dejaco C, Dragosics B, Mosberger I, Mayr W, et al.. Blood groups Lewis (b) and $\mathrm{ABH}$ expression in gastric mucosa: lack of inter-relation with Helicobacter pylori colonisation and occurrence of gastric MALT Iymphoma. Gut. 1997;41(1):37-42.

6. Suzuki T, Matsuo K, Ito H, Hirose K, Wakai K, Saito T, et. al. A past history of gastric ulcers and Helicobacter pylori infection increase the risk of gastric malignan lymphoma. Carcinogenesis 2006;27(7):1391-7.

7. Zinkiewics K, Dzierzanowska D, Chibowski D, Ligieza J, Wallner G, Sory A, et al. Topography of Helicobacter pylori infection in gastric cancer patients. J Physiol Pharmacol. 1997;48(4):133-8.
8. World Health Organization (WHO), International Agency for Research on Cancer Liver flukes and Helicobacter pylori: IARC monographs on the evaluation of the carcinogenic risks to humans. Schistosomes, Liver Flukes and Helicobacter pylori. Lyon: WHO; 1994.

9. Debongnie J, Pauwels S, Raat A, De Meius Y, Haot J, Mainguet P. Quantification of Helicobacter pylori infection in gastritis and ulcer disease with a simple an rapid carbon-14urea breath test. J Nucl Med 1991;32(6):1192-8.

10. Kosunen T, Seppala K, Sarna S, Sipponen $\mathbf{P}$. Diagnostic value of decreasing $\lg G$, IgA and IgM antibody titres after eradication of Helicobacter pylori. Lancet 1992;339(8798):893-5.

11. Lage A, Fauconnier A, Burette A, Glupczynski Y. Rapid colorimetric hybridization assay for detecting amplified Helicobacter pylori DNA in gastric biopsy specimens. J Clin Microbiol. 1996;34(3):530-3.

12. Lu J, Perng C, Shyu R, Chen C. Comparison of five PCR methods for detection of Helicobacter pylori DNA in gastric tissues. J Clin Microbiol. 1999;37(3):772-4.

13. Ni Y, Lin J, Huang S, Yang J, Chang M. Accurate diagnostic of Helicobacter pylori infection by stool antigen test and other currently available test in children. J Pediatr. 2000;136(6):823-7.

14. Premoli G, Gonzáles A, Millán-Mendoza B, Percoco T, Vielma A. Diagnóstico de Helicobacter pylori mediante la reacción en cadena de la polimerasa. Rev Cubana Med Trop. 2004;56(2):85-90.

15. Marais A, Monteiro L, Occhialini A, Pina M, Lamouliatte H, Megraud F. Direct detection of Helicobacter pylori resistance to macrolides by a polymerase chain reaction/ DNA enzyme immunoassay in gastric biopsy specimens. Gut 1999;44(4):463-7.

16. Monteiro L, Cabrita J, Megraud F. Evaluation of performances of three DNA-Enzyme immunoassays for detection of Helicobacter pylori PCR products from biopsy specimens. J Clin Microbiol. 1997;35(11):2931-6.

17. Ramirez-Ramos A, Mendoza-Requena D, Leey-Casella J, Guerra-Valencia J. Estudio del Helicobacter pylori en el Perú. Rev Per Med Exp Salúd Pública. 2002;19(4):209-214.

18. Ramírez-Ramos A, Recavarren-Arce $S$, Arias-Stella J, Gilman RH, León-Barúa R, Garrido-Klinge G, et. al. Helicobacter pylori, Gastritis Cónica, Ulcera Gástrica y Ulcera Duodenal: Estudio de 1638 pacientes. Rev Gastroenterol Perú. 1999;19(3):196-201.

19. Rivera E, López-Vidal Y, Luqueño V, Ruiz-Palacios GM. Indirect immunofluorescence assay for detection of Helicobacter pylori in human gastric mucosal biopsies. J. Clin. Microbiol. 1991;29(8):1748-51.

20. Velapatiño B, Balqui J, Gelman RH, Bussalleu A, Quino W, Finger SA, et. al. Validation of String Test for Diagnosis of Helicobacter pylori Infections. J. Clin. Microbiol. 2006;44(3):976-80.

Correspondencia: César Tadeo Vela-Velásquez. Dirección: Jr. Colina 1081, Callao 2, Lima, Perú.

Correo electrónico: citopatperu@yahoo.com 